\title{
PREDICTION OF SWELLING PARAMETERS OF TWO CLAYEY SOILS FROM ALGERIA USING ARTIFICIAL NEURAL NETWORKS
}

FATIMA ZOHRA MEROUANE - PhD Student, University Center of Ain Temouchent, Departement of civil engineering, e-mail: merouanefatima@hotmail.fr

SIDI MOHAMED AISSA MAMOUNE - Lecturer, PhD, University Center of Ain Temouchent, Departement of civil engineering, e-mail: aissa_mamoune@yahoo.fr

\begin{abstract}
The phenomenon of swelling is one of the more complicated geotechnical problems that the engineer have to deal with. However, its quantification is essential for the design of structures and various methods can be applied to the identification of this phenomenon. Some, such as mineralogical identification and direct measurements of swelling, are more or less long and require very specific equipment. However, there are other methods that offer the advantage of being relatively fast and lesser expensive: they are based on soil mechanics parameters. Using these parameters, several authors have introduced soil swelling prediction models, mostly in the form of classifications and empirical formulas. This work concerns in the first part the identification and classification of the swelling potential of two clays located in north-western Algeria. Followed by a statistical analysis carried out to test the reliability of the observations for the estimation of the pressure and the swelling amplitude using a multiple linear regression.

A second part is devoted to the development of a prediction method by artificial neural networks allowing the estimation of swelling parameters (pressure and amplitude) by minimizing the difference between the experimental measurements and the numerical results. Modeling by artificial neural networks is of great interest in the field of prediction. The application of two networks makes it possible to obtain good forecasts of the swelling parameters.
\end{abstract}

Keywords: swelling; pressure-amplitude; estimation; multiple linear regression; neural networks

\section{Introduction}

Swelling parameters such as pressure and amplitude are the most important soil parameters that are used for design in geotechnical engineering work. Having the ability to prevent the swelling of the soil during the reconnaissance campaign would greatly assist the engineers in their choice of foundation type.

However, estimating the behaviour of expansive soils is very complex. To overcome with these complications, several approaches have been developed by researchers. Apart from the long and expensive direct methods of identification, statistical methods have been characterized by swelling soils since the 1960 s.

A review of the literature reveals a significant number of empirical approaches developed for identifying and characterizing the swelling phenomenon.

The most used are: Altmeyer [1]; Seed et al. [2]; Ranganatliam and Satyanarayana [3]; Komornik and David [4]; Vijayvergiya and Ghazzaly [5]; Dakshanamurthy and Raman [6]; Williams and Donaldson [7]; the Building Research Establishment [8]; Snethen [9]; Chen [10]. Other researchers have tried to provide additional information on the behavior of swelling soils by establishing new models, Basma A.A [11]; Al-Shayea N.A [12]; Iş1k Yilmaz [13] and Djedid A. et al. [14]; Ömür Ç. et al. [15].

These models could allow us to estimate the behaviour of swelling soils according to physicochemical identification parameters that are simple to implement, such as Atterberg limits and particle size.

The development or adjustment of such models requires a prior knowledge of the nature of the relationships between the data whose behaviour depends on many external factors of composition 
and environment. The interactions of these factors are difficult to establish solely by traditional statistics methods because of their interdependence.

Given the complexity of the phenomenon, the process to follow becomes a little more complex, modelling through powerful numerical techniques of this phenomenon is a good approach to solve this problem and obtain better results.

One of these techniques is the artificial neural network (ANNs), which presents a form of artificial intelligence; it is a simplified mathematical model of the biological nervous system. ANNs learn examples of data presented to them to capture the subtle functional relationships between the data and allow to integrate the nonlinear behaviour of materials in the analysis of soil systems that present extreme variability. ANNs are therefore well suited to modelling the complex behaviour of the swelling phenomenon.

The use of ANNs in recent years has been increased in all science and technology disciplines. Also, ANNs have been successfully applied to many geotechnical engineering applications.

Many researchers have used ANNs for modelling the behaviour and solve problems related to swelling soils. Najjar YM and Basheer IA [16-17] used ANNs to model swelling and concluded that the ANNs technique performed better than multivariate regression analysis. Basma AA et al. [18], model the amplitude of swelling of a clay as a function of time using ANNs. Meisina $\mathrm{C}$ and Najjar Y [19], used ANNs to estimate the shrinkage-swelling of an Italian clay, and the results were better than traditional mathematical modelling, showing the effectiveness of the ANNs technique. Moosavi $\mathrm{M}$ et al. [20], modelled the cyclic swelling pressure of sludge rock, using ANNs. Erzin, Y. [21] investigated the behaviour of swelling pressure as a function of soil suction, using artificial neural networks. Doris JJ et al. [22] used ANNs for the prediction of vertical surface movement of soils due to shrinkage-swelling. Bekkouche A [23], Ashayeri I et al. [24] and Aissa Mamoune SM [25] estimated the amplitude and swelling pressure of unsaturated clays using ANNs. Banu Ikizler S et al. [26], also estimated the swelling pressure of expansive soils with ANNs.

The growing interest of geotechnical engineers in neural networks is due to their excellent ability to model nonlinear problems with multiple variable. Neural networks can capture nonlinear interactions between variables and produce reasonably accurate forecasts. Compared to other conventional methods, this has the advantage of being immediately updated as new results are accumulated.

This article consists of two parts. The objective of the first part is to estimate the swelling parameters, using statistical analysis, in particular multiple linear regression analysis. This study presents the application of a method of multiple analysis for the estimation of the swelling of two clays located in north-western Algeria from physicochemical identification parameters determined in the laboratory. Correlation equations obtained from multiple regression analyses have been shown to be reliable in practical situations.

In the second part, Artificial Neural Networks (ANNs) are used to predict the pressure and the swelling amplitude of the two clays from two different sites in north-western Algeria. To do this, two ANNs models have been developed to predict swelling parameters using experimental geotechnical identification results. It has been found that the predicted values are quite close to the calculated values. In addition, two performance indices such as the coefficient of determination and the mean squared error were calculated to check the prediction capacity and accuracy of the developed ANNs models. Built ANNs models have shown high predictive performance and indicate that there are strong correlations between soil properties and swelling parameters. The results demonstrate that the developed ANNs models can be used at the preliminary design stage. 


\section{Geographical and geological situation of the studied soils}

Our study was carried out on two clays of north-western Algeria, the first is from Mascara (northwest of Algeria) (Fig. 1) in an active area of swelling. It comes from the site of the project of realization of penetrating, connecting SIG with the east-west highway. The second clay is located in the group Chetouane Mansourah Tlemcen (extreme northwest of Algeria). The two soils are made of the marly clays of the Miocene basin.

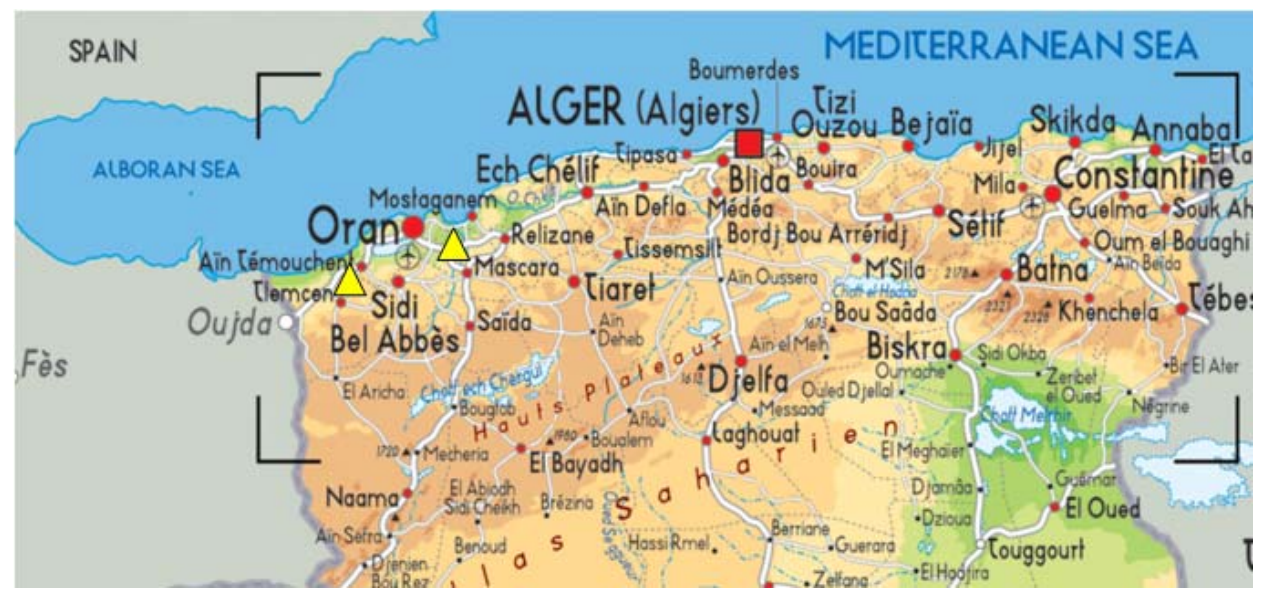

Fig. 1 - Geographical situation of the studied soils

\section{Identification of the swelling of the studied soils}

In the preliminary reconnaissance phase, the identification of the swelling potential of a given soil is essential; it will enable the engineer to choose the foundation type to be adopted and guides him towards the tests to be carried out during the thorough reconnaissance.

In Algeria, several authors have tested methods to estimate the swelling potential at several local sites [14, 27-30]. The application of these methods generally confirms the qualitative prediction of swelling.

\subsection{Qualitative identification of swelling}

In order to identify the swelling potential of soils, several authors have linked it to one or more elementary geotechnical identification parameters, as proposed by Seed et al. [2]; Snethen [9]; the Building Research Establishment [8] and Chen [10]; and as shown in Table 1, These classifications are based on the percentage of the clay fraction, the liquid limit and the plasticity index.

Table 1

Empirical approaches between physicochemical characteristics and swelling

\begin{tabular}{|c|c|c|c|c|c|c|}
\hline \multicolumn{3}{|c|}{ Seed, Woodward and Lungreen (1962) } & \multicolumn{4}{|c|}{ Snethen (1980) } \\
\hline Swelling Potential & $\mathrm{Sp}(\%)$ & PI $(\%)$ & Swelling $\mathrm{F}$ & ential & & \\
\hline \multirow{2}{*}{ Low } & \multirow{2}{*}{$0-1.5$} & \multirow{2}{*}{$0-10$} & \multicolumn{2}{|c|}{ Very High } & \multicolumn{2}{|c|}{$>35$} \\
\hline & & & \multicolumn{2}{|c|}{ High } & \multicolumn{2}{|c|}{$22-48$} \\
\hline Medium & $1.5-5$ & $10-20$ & \multicolumn{2}{|c|}{ Medium } & \multicolumn{2}{|c|}{$22-32$} \\
\hline High & $5-25$ & $20-35$ & \multicolumn{2}{|c|}{ Low } & \multicolumn{2}{|c|}{$<18$} \\
\hline \multicolumn{3}{|c|}{ BRE (1980) } & \multicolumn{4}{|c|}{ Chen (1988) } \\
\hline PI $(\%)$ & $\mathrm{C}(\%)<2 \mu \mathrm{m}$ & Swelling Potential & $\mathrm{C}(\%)<74 \mu \mathrm{m}$ & $\operatorname{LL}(\%)$ & $\begin{array}{c}\text { Swelling } \\
\text { Pressure } \\
\text { (bars) }\end{array}$ & $\begin{array}{l}\text { Swelling } \\
\text { Potential }\end{array}$ \\
\hline$>35$ & $>95$ & Very High & $>95$ & $>60$ & 10 & Very High \\
\hline $22-35$ & $60-95$ & High & $60-95$ & $40-60$ & $2.5-5$ & High \\
\hline $18-22$ & $30-60$ & Medium & $30-60$ & $30-40$ & $1.5-2.5$ & Medium \\
\hline$<18$ & $<30$ & Low & $<30$ & $<30$ & $<0.5$ & Low \\
\hline
\end{tabular}


Other authors use abacus to classify swelling soils.

Seed et al (1962), Williams et Donaldson (1980), proposed a method for estimating the swelling by reference to soil clay content and activity. Vijayvergiya and Ghazzaly, Dakshanamurphy and Raman (1973), use the Casagrande diagram. On this abacus are placed the representative points of the soil samples of Mascara and Tlemcen. (Fig. 2).
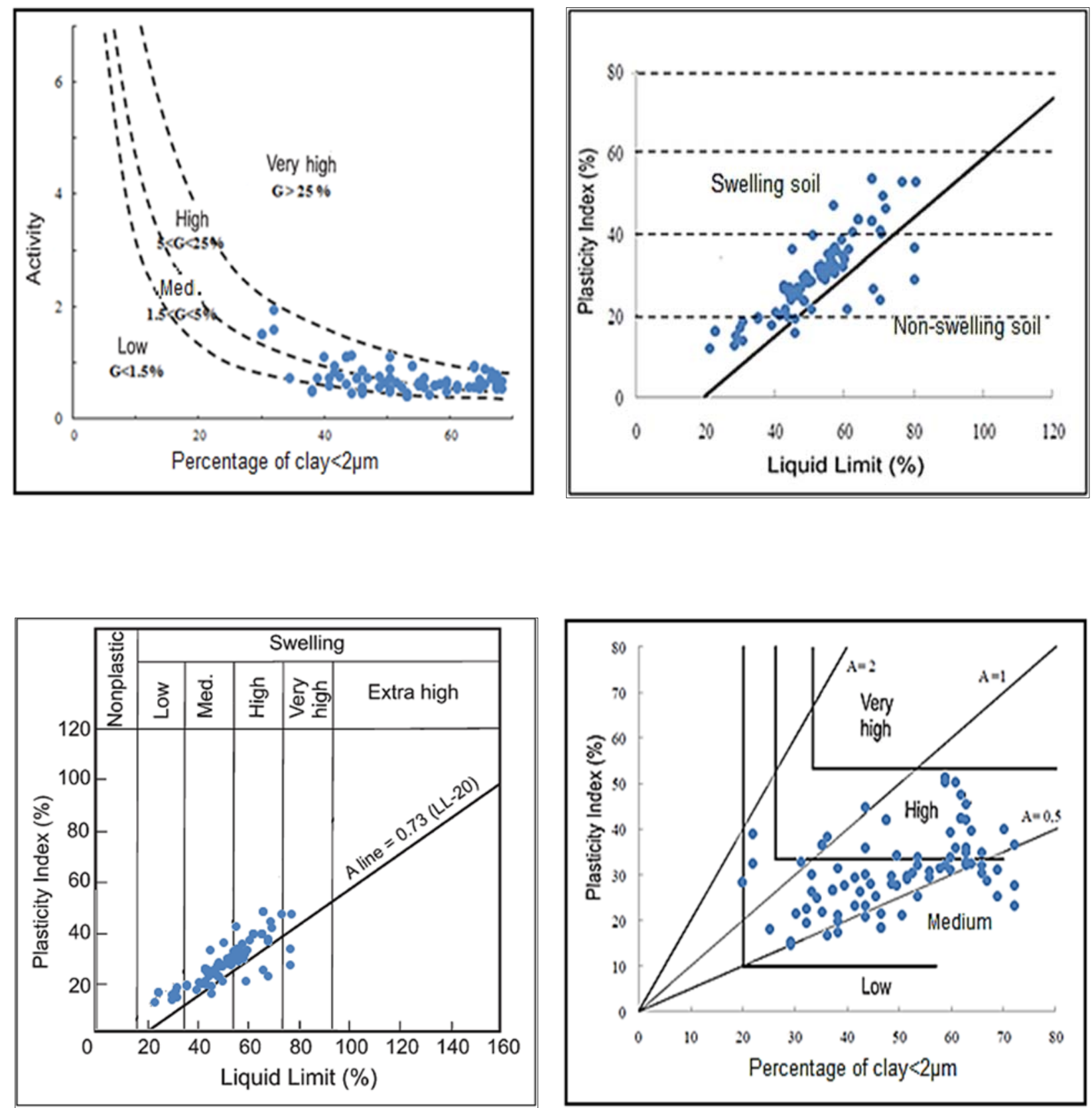

Fig. 2 - Determination of the swelling potential using the different classifications

These classifications were applied to our clays, and according to the available geotechnical identification parameters, these indirect estimations all revealed the existence of the risk of swelling for the studied soils.

The Table 2 summarizes the results of the various estimation methods used to classify the swelling potential of the studied clays. 
Swelling potential of soils examined by different classifications

\begin{tabular}{|c|c|c|c|c|c|c|}
\cline { 2 - 6 } \multicolumn{1}{c|}{} & $\begin{array}{c}\text { Seed, } \\
\text { Woodward } \\
\text { and } \\
\text { Lungreen }\end{array}$ & Snethen & $\begin{array}{c}\text { The Building } \\
\text { Research } \\
\text { Establishment }\end{array}$ & Chen & $\begin{array}{c}\text { Dakshanamurphy } \\
\text { and Raman }\end{array}$ & $\begin{array}{c}\text { Williams } \\
\text { and } \\
\text { Donaldson }\end{array}$ \\
\hline $\begin{array}{c}\text { Clays of } \\
\text { Mascara } \\
\text { and } \\
\text { Tlemcen }\end{array}$ & $\begin{array}{c}\text { High and } \\
\text { medium to } \\
\text { high }\end{array}$ & $\begin{array}{c}\text { Medium } \\
\text { to High }\end{array}$ & High & High & $\begin{array}{c}\text { Medium } \\
\text { to High }\end{array}$ & $\begin{array}{c}\text { Medium } \\
\text { to High }\end{array}$ \\
\hline
\end{tabular}

\subsection{Quantitative estimation of soil swelling parameters}

From the same geotechnical parameters used in the classification of swelling soils, an estimation of the swelling parameters (Pressure and Amplitude) is possible by using the empirical correlations proposed in the literature.

To appreciate the reliability of these models, they were applied to clays of Tlemcen and Mascara.

A large discrepancy between the estimation results obtained is noted (Table 3). As a result, these prediction models are inapplicable for estimating the swelling parameters of the studied soils. Even adjusted, the difference between the estimated values and those measured remains very high.

Table 3

Results of adjusted swelling models

\begin{tabular}{|c|c|c|c|}
\hline Models & $\begin{array}{l}\text { Mathematical } \\
\text { Expressions }\end{array}$ & $\begin{array}{c}\mathbf{R}^{2} \text { (Between } \\
\text { estimated and measured }\end{array}$ & $\mathbf{R}^{2}$ (Adjusted models) \\
\hline Seed et al. (1962) [2] & $S p=2.16 \times 10^{-3} \times P I^{2.44}$ & 0.077 & 0.081 \\
\hline \multirow{2}{*}{$\begin{array}{l}\text { Komornik and David } \\
\text { (1969) [4] }\end{array}$} & $S p=0.9+2.1 \times P I$ & 0.081 & 0.081 \\
\hline & $\begin{array}{r}\log P s=-2.132+ \\
0.0208 x\end{array}$ & 0.077 & 0.086 \\
\hline \multirow{2}{*}{$\begin{array}{c}\text { Nayak and Christensen } \\
\text { (1971) }[31]\end{array}$} & $\begin{array}{c}S p=2.29 \times 10^{-2} \times P I^{1.45} x \\
C / W+6.39\end{array}$ & 0.089 & 0.092 \\
\hline & $\begin{array}{c}P S=3.5817 \times 10^{-2} x \\
P I^{1.12} \times C^{2} / W^{2}+3.7912\end{array}$ & 0.004 & 0.067 \\
\hline \multirow{2}{*}{$\begin{array}{l}\text { Vijayvergiya and } \\
\text { Ghazali (1973) [5] }\end{array}$} & $\begin{array}{c}\log S p=(0.4 x L L-W+ \\
5.5) / 12\end{array}$ & 0.016 & 0.016 \\
\hline & $\begin{array}{c}\log P S=(0.4 x L L-W+ \\
23.6) / 12\end{array}$ & 0.085 & 0.025 \\
\hline Weston D.J (1980) [32] & $S p=2 / 3 \times 10^{(0.9 \times P I / W-}$ & 0.048 & 0.095 \\
\hline Chen F.H (1988) [10] & $S p=\underset{P I}{0.2558 x(e)^{0.08381 x}}$ & 0.067 & 0.080 \\
\hline Basma A.A (1993) [11] & $\begin{array}{c}S p=6.4 x 10^{-4} \times P I^{1.37} x \\
C^{1.37}\end{array}$ & 0.080 & 0.082 \\
\hline Al-Shayea N.A (2001) [12] & $S p=0.55 \times C-8.25$ & 0.031 & 0.031 \\
\hline
\end{tabular}




\section{Multiple linear regression (MLR)}

In order to better estimate the swelling character of the two clays, a regression analysis was carried out to search for more significant parameters and develop new swelling prediction models specific to the studied clays, which will be more reliable with the minimum of errors.

The general form of the theoretical model of multiple linear regression is written as follows [33]:

with:

$$
\mathrm{Y}=\mathrm{A}_{0}+\mathrm{A}_{1} \mathrm{X}_{1}+\mathrm{A}_{2} \mathrm{X}_{2}+\ldots+\mathrm{A}_{\mathrm{n}} \mathrm{X}_{\mathrm{n}}+\varepsilon
$$

$Y$ - Dependent variable (explained);

$\mathrm{X}_{1}, \ldots \mathrm{X}_{\mathrm{n}}$ - Independent variable (predictor);

$\mathrm{A}_{0}, \ldots \mathrm{A}_{\mathrm{n}}$ - Model parameters estimated using the least squares method;

$\varepsilon$ - Residue.

The quality of fit is assessed using the correlation coefficient $\mathrm{R}$.

\subsection{Statistical analysis of geotechnical data}

For a good estimation of the pressure and the amplitude of swelling of the studied clays, a proper choice of factors is necessary; for that, the elaboration of a data base including the elementary parameters relating to the studied soils was made.

Then a statistical analysis on the available data was carried out in order to test the representativeness by variable to look for specific models to these soils.

The following Table 4 represents the global population studied, where Z, C, W, LL, PI, A and $\mathrm{Y}_{\mathrm{d}}$ respectively represent the depth (in $\mathrm{m}$ ), the clay content (in\%), the natural water content (in\%), the liquid limit (in\%), the plasticity index (in\%), the activity, the dry density (in $\mathrm{kN} / \mathrm{m}^{3}$ ), the swelling pressure (in bars) and the swelling amplitude (in \%).

Table 4

\begin{tabular}{|c|c|c|c|c|c|c|c|c|c|c|c|}
\cline { 2 - 13 } \multicolumn{1}{c|}{} & $\mathbf{Z}$ & $\mathbf{C}$ & $\mathbf{W}$ & $\mathbf{L L}$ & $\mathbf{P I}$ & $\mathbf{A}$ & $\mathrm{V}_{\mathbf{d}}$ & $\mathbf{P s}$ & $\begin{array}{c}\text { Log } \\
\text { Ps }\end{array}$ & $\mathbf{S p}$ & $\begin{array}{c}\text { Log } \\
\text { Sp }\end{array}$ \\
\hline $\begin{array}{c}\text { Nbr } \\
\text { Observations }\end{array}$ & 90 & 90 & 90 & 90 & 90 & 90 & 90 & 90 & 90 & 65 & 65 \\
\hline Minimum & 1.00 & 28.00 & 12.09 & 34.50 & 22.00 & 0.39 & 1.29 & 0.6 & -0.22 & 0.15 & -0.10 \\
\hline Maximum & 11.80 & 79.00 & 34.17 & 90.40 & 60.36 & 1.58 & 2.05 & 8.88 & 0.95 & 15.2 & 1.18 \\
\hline Mean & 4.66 & 59.34 & 21.09 & 64.27 & 39.33 & 0.72 & 1.71 & 2.79 & 0.36 & 5.52 & 0.63 \\
\hline $\begin{array}{c}\text { Standard } \\
\text { deviation }\end{array}$ & 3.09 & 13.36 & 5.13 & 12.16 & 8.92 & 0.20 & 0.15 & 1.69 & 0.27 & 3.91 & 0.35 \\
\hline $\begin{array}{c}\text { Coef. } \\
\text { Variation }\end{array}$ & $66.42 \%$ & $23.72 \%$ & $24.34 \%$ & $18.92 \%$ & $22.68 \%$ & $28.79 \%$ & $9.19 \%$ & $60.61 \%$ & $74.23 \%$ & $70.78 \% 55.70 \%$ \\
\hline
\end{tabular}

The analysis of this table gives us an idea about the dispersion of the studied parameters. The pressure and the amplitude of swelling are the parameters which vary the most with high coefficients of variation ( $60.61 \%$ and $70.78 \%$ respectively). Some parameters are more or less scattered such as: the clay fraction (coefficients of variation: $23.72 \%$ ), the activity $(28.79 \%$ ) and the natural water content $(24.34 \%)$, which explains the difficulty of determining the swelling parameters compared to other identification parameters.

Besides, we note that the average percentage of fines is $59.34 \%(>50 \%)$, which indicates a significant presence of clay in the studied soils. 
Based on the statistical analysis and according to the R-adjusted graphs (Figs. 3 and 5), the models given below have been developed; they are considered best appropriate for the prediction of swelling pressure (Table 5) and amplitude swelling (Table 6) of studied clays.

\subsection{Proposed models for swelling pressure}

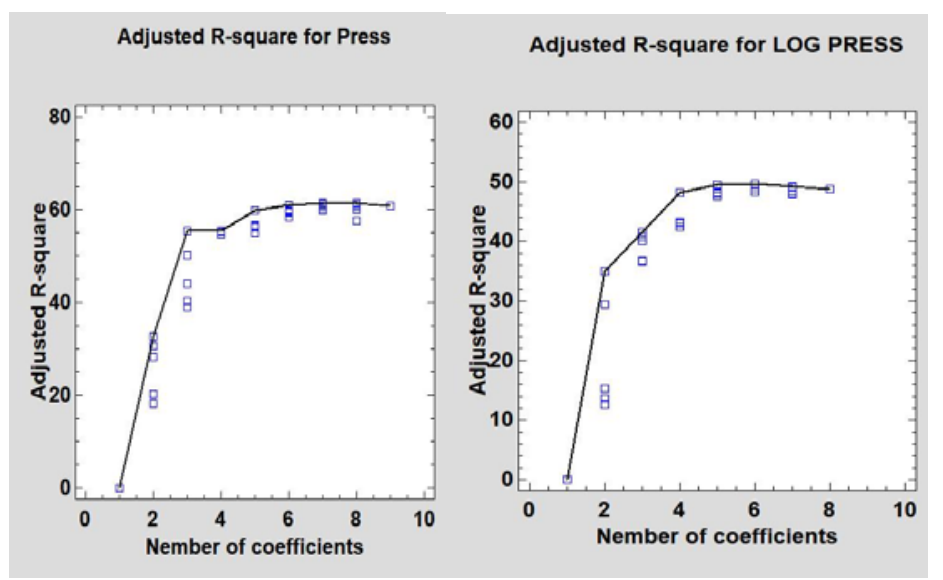

Fig. 3 - R-squared Variations depending on the number of Variables for the swelling pressure

The $\mathrm{R}^{2}$ adjusted pressure and amplitude graphs give the number of coefficients to be used for model development based on the R-adjusted. In our case, we note that for the two parameters of swelling (pressure and amplitude), the use of at least four coefficients would be enough to obtain better correlations (Figs. 3 and 5).

Table 5

Proposed models for the swelling pressure

\begin{tabular}{|c|c|c|}
\hline $\mathbf{N}^{\circ}$ & MLR Models & Results \\
\hline 1 & $\begin{array}{c}P s=-5.599-0.153 \times Z+0.034 \times C+0.145 \times W+ \\
2.374 \times V_{d}\end{array}$ & $\mathbf{R}^{2}=41.81 \%$ \\
\hline 2 & $\begin{array}{c}\log P s=-0.520-0.023 \times Z+0.023 \times W+0.010 \times P I \\
-0.384 \times A+0.214 \times V_{d}\end{array}$ & $\mathbf{R}^{2}=52.46 \%$ \\
\hline
\end{tabular}

For the swelling pressure, the two models developed give remarkable differences between measurements and calculations with $\mathrm{R}^{2}=41.81 \%$ for Ps and $\mathrm{R}^{2}=52.46 \%$ for Log Ps (Table 5), (Figs. 4 (1)-(2)).

Similarly, for amplitude, the $\mathrm{R}^{2}$ varies from $58.04 \%$ to $57.78 \%$ for $\mathrm{Sp}$ and Log Sp respectively (Table 6), (Figs. 6 (1)-(2)).

The reason is that the model estimates the pressure for two different clays at the same time. This makes modelling more complex, as well as other parameters not considered in the calculations, such as the grain size of the soil [34], the nature of clay minerals, the shape of the particles, and their structural arrangement, which can significantly affect soil swelling.
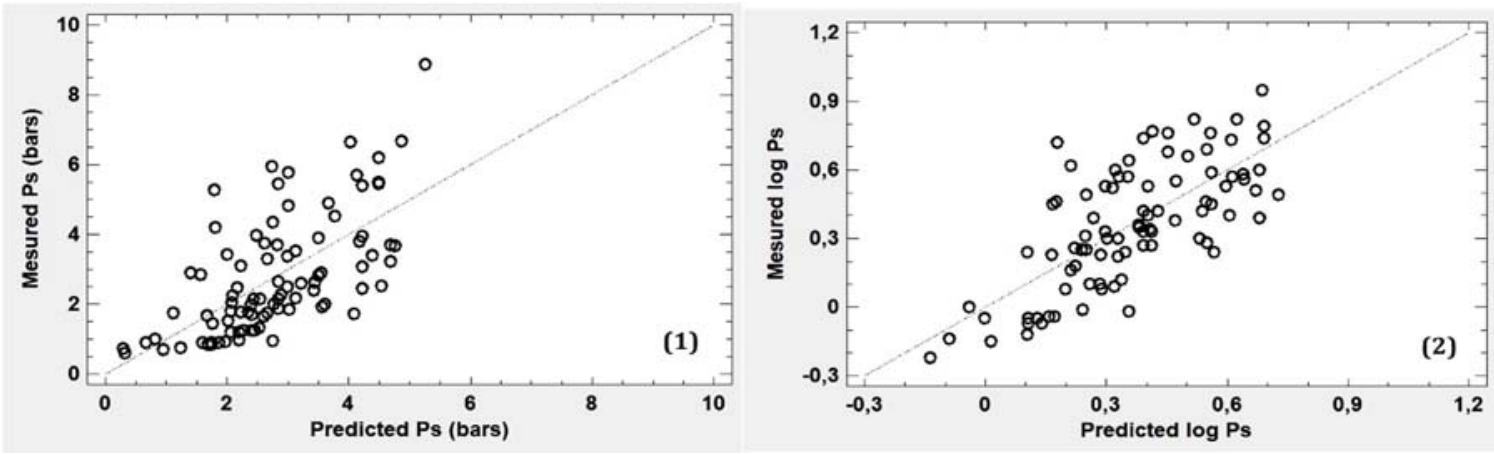

Fig. 4 - The cross-correlation between predicted and measured values of swelling pressure 


\subsection{Proposed models for swelling amplitude}

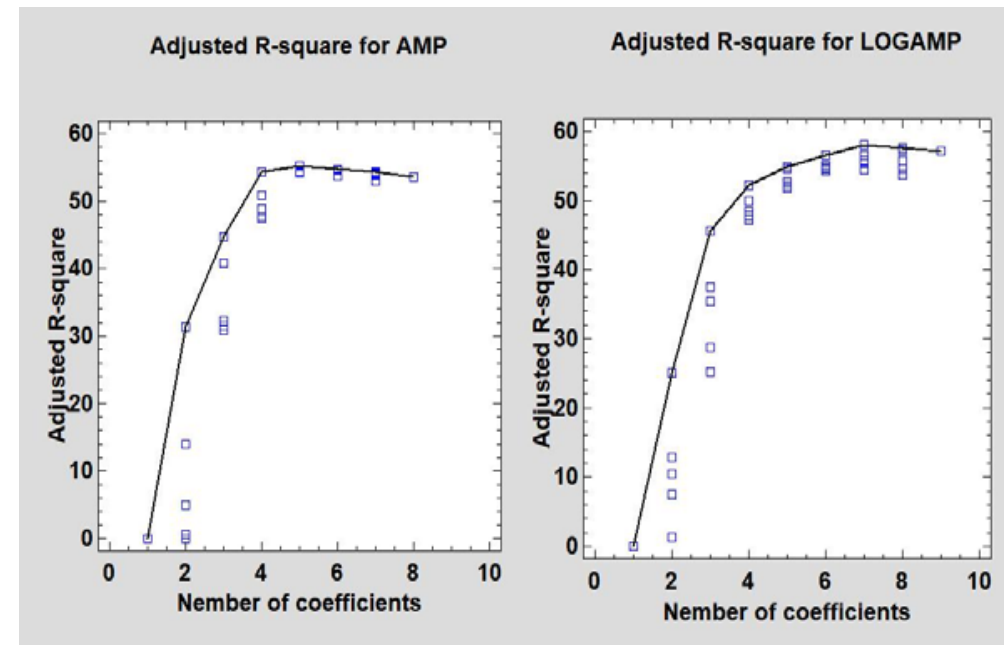

Fig. 5 - R-squared Variations depending on the number of Variables for the swelling amplitude

Table 6

Proposed models for the swelling amplitude

\begin{tabular}{|c|c|c|}
\hline $\mathbf{N}^{\circ}$ & MLR Models & Results \\
\hline $\mathbf{1}$ & $\boldsymbol{S p}=-3.978-0.775 \times \begin{array}{c}0.185 \times C-0.258 \times \mathrm{W}+ \\
4.169 \times \mathrm{Vd}\end{array}$ & $\mathbf{R}^{\mathbf{2}}=58.04 \%$ \\
\hline $\mathbf{2}$ & $\begin{array}{c}\text { Log } \boldsymbol{S p}=-0.493-0.066 \times Z+0.017 \times C-0.026 \times \mathrm{W}+ \\
0.533 \times \mathrm{Vd}\end{array}$ & $\mathbf{R}^{\mathbf{2}}=57.78 \%$ \\
\hline
\end{tabular}
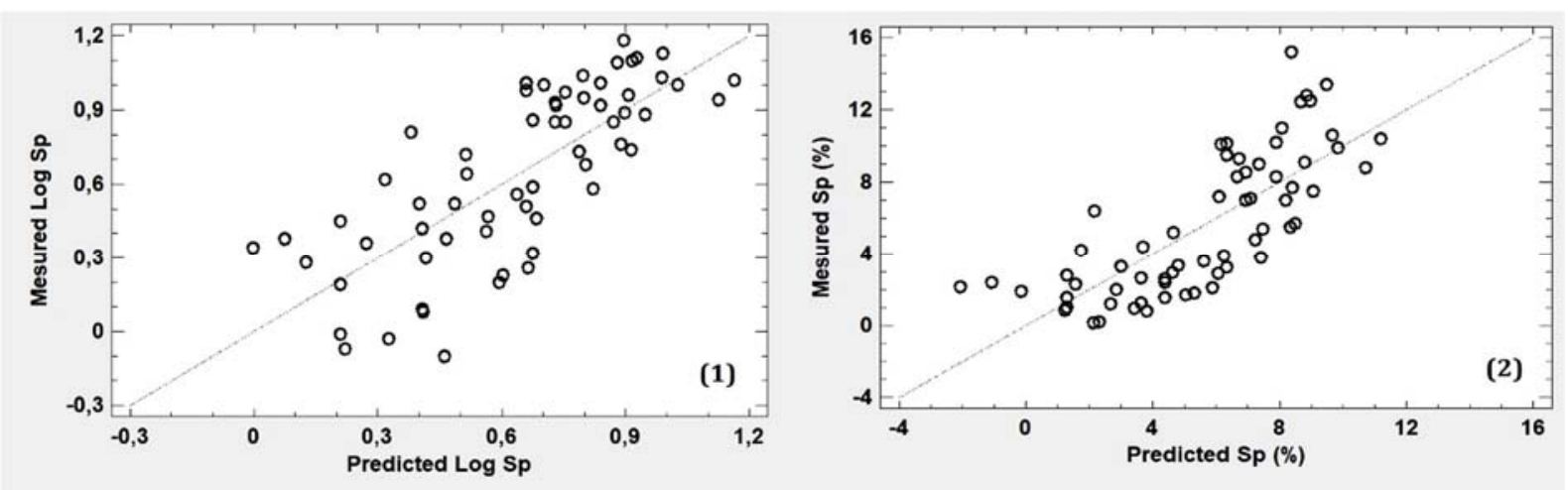

Fig. 6 - The cross-correlation between predicted and measured values of swelling amplitude

The multiple regression modelling of the forecast model coefficients allowed a slight improvement in the computational results.

Given the observed discrepancies between the observed swellings and the results of the statistical calculations, and because of the complexity of the swelling phenomenon, non-linear regression analysis was performed using artificial neural networks to better adjust the models to the studied clays. This analysis aimed to find new prediction models with optimal values of correlation coefficients between measurements and calculations.

\section{Artificial neural networks}

Artificial neural networks are mathematical models composed of strongly connected nodes. They develop a complex input model with a complex output model [35-36]. The power of neural calculus comes from the massive intercommunication of neurons and the adaptive nature of the 
weights that connect all neurons. These have proved very useful in learning complex relationships between multidimensional data.

There are several types of networks, the latter differ essentially in the architecture and rules of learning and organization. The most commonly used networks are multilayer perceptrons qualified by inverse propagation algorithms. These are simple, robust and very powerful in identifying the model and classification.

\subsection{The artificial neuron}

The architecture of a neural network and the structure of an artificial neuron are represented in Fig. 7. The input values $\left(\mathrm{x}_{1}, \mathrm{x}_{2}, \ldots, \mathrm{Xn}_{\mathrm{n}}\right)$ and the weights associated with the inputs $\left(\mathrm{W}_{\mathrm{j} 1}, \mathrm{Wj}_{\mathrm{j} 2}, \ldots, \mathrm{Wjn}_{\mathrm{j}}\right)$ are the variables of the activation function that determines the activation value of the neuron. This activation value is then passed as an argument to the output function, which determines the output value of the neuron, $\mathrm{y}_{\mathrm{j}}$. The additional input (y) is used to indicate to the neuron the expected output value so that the neuron can correct its weight to approach this value.

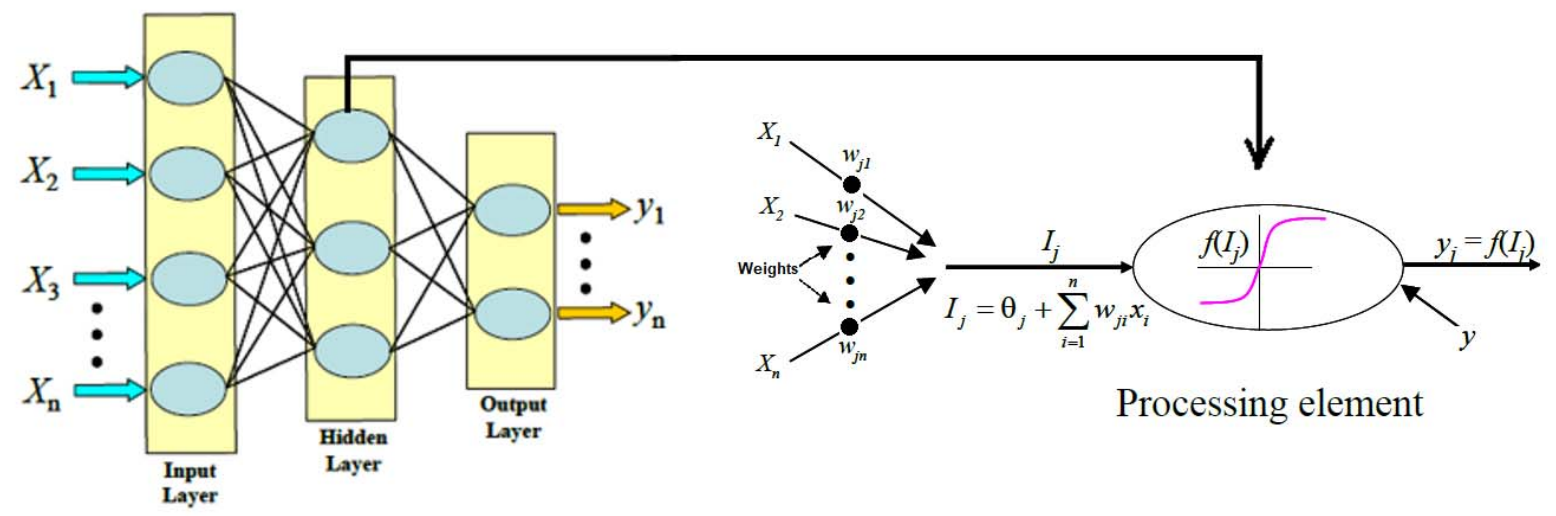

Fig. 7 - Typical structure and operation of ANNs [37]

The result of this combined summation is passed through a transfer function (e.g. logistic sigmoid or hyperbolic tangent) to produce the output of the processing element. For node $\mathrm{j}$, this process is summarized in Equation 2 as illustrated in Fig. 7.

$$
I j=\theta j+\sum_{i=1}^{n} W j i x j
$$

where

$I_{j}$ - the activation level of node $j$;

$\mathrm{W}_{\mathrm{ji}}$ - the connection weight between nodes $\mathrm{j}$ and $\mathrm{i}$;

$\mathrm{X}_{\mathrm{i}}$ - the input from node $\mathrm{i}, \mathrm{i}=0,1, \ldots, \mathrm{n}$;

$\theta_{\mathrm{j}}$ - the bias or threshold for node $\mathrm{j}$;

$Y_{j}$ - the output of node $j$; and

$f\left(I_{j}\right)$ - the transfer function.

In this work, we use the Levenberg-Marquardt error backpropagation algorithm with networks of 3 to 8 layers of neurons. The transfer function applied is of sigmoid type (Equation 3).

$$
f\left(I_{J}\right)=\frac{1}{1+e^{-\alpha\left(I_{J}\right)}}
$$

where: $\alpha$ is a constant used to control the slope of the semi-linear region. This function transforms the interval $[-\infty ;+\infty]$ to $[-1 ;+1]$. 


\subsection{Training of developed models}

Two prediction models were developed ANN 1 to predict swelling pressure, ANN 2 for swelling amplitude. To develop these models, we presented to the input networks the values of seven calculated geotechnical identification parameters of the two clays, these parameters are, the depth, the percentage of the clay fraction, the natural water content, the limit of liquidity, the plasticity index, the activity and dry density. The pressure and the swelling amplitude represent the output parameters.

A first step is to test the models developed with the learning data, then to validate these models, we introduce in the model samples that were not considered during the learning process. In this work 90 sets of data from both Mascara and Tlemcen clays were used for the swelling pressure and 65 for the swelling amplitude, $70 \%$ of the data were chosen for training and the remaining $30 \%$ were selected as a validation and testing set. The network structure is optimized to obtain a good forecasting capacity (Table 7).

During training, an evaluation of network performance is done as they try to find the optimal weight set. The mean square errors (MSE) (Equation 4) and the correlation coefficient (R) (Equation 5) are calculated.

Mean Squared Error is the average squared difference between outputs and targets. Lower values are better. Zero means no error.

Regression $\mathrm{R}$ Values measure the correlation between outputs and targets. An R coefficient value close to 1 , means a better estimation.

$$
\begin{gathered}
M S E=\frac{1}{N} \sum_{j=1}^{N}\left(Y-Y_{j}\right)^{2} \\
R=\frac{\sum_{j=1}^{N}(Y-\bar{Y})\left(Y_{j}-\overline{Y_{j}}\right)}{\sqrt{\sum_{j=1}^{N}(Y-\bar{Y})^{2}} \sqrt{\sum_{i=1}^{N}\left(Y_{j}-\overline{Y_{j}}\right)^{2}}}
\end{gathered}
$$

Where

$\mathrm{Y}, \mathrm{Y}_{\mathrm{j}}$ - denote the observed values and predicted values by ANN;

$\bar{Y}, \overline{Y_{j}}$ - are the mean of $Y, Y_{j}$ respectively;

$\mathrm{N}$ - represents the number of data.

Table 7

Parameters of the various developed models by ANN

\begin{tabular}{|c|c|c|c|}
\hline Networks & Number of INPUTS & Hidden Layer & $\begin{array}{c}\text { Number of } \\
\text { OUTPUTS }\end{array}$ \\
\hline ANN 1 $(\mathrm{Ps})$ & $7 \times 90$ & 8 & $1 \times 90$ \\
\hline ANN 2 $(\log \mathrm{Sp})$ & $7 \times 65$ & 3 & $1 \times 65$ \\
\hline
\end{tabular}

The results of the two models ANN 1, ANN 2 are plotted in Figs. 8 - 10 respectively, where the measurements are plotted on the abscissa and the estimated values by the different models on the ordinate.

The errors results between targets and outputs by ANN's models are given in figures 9 and 11 for swelling pressure and swelling amplitude respectively.

In the training phase, a better correlation between measured and estimated swelling parameters has been obtained (Table 8). For the swelling pressure, the ANN 1 estimates a high correlation coefficient (R) of 0.96 with a low mean square error (MSE) of 4.79. For swelling amplitude, the ANN 2 estimates a correlation coefficient (R) of 0.95 with an MSE of 0.018. 
Performance results of the developed Models

\begin{tabular}{|c|c|c|c|c|c|c|}
\hline \multirow{2}{*}{ Networks } & \multicolumn{2}{|c|}{ ANN 1 (Swelling pressure) } & \multicolumn{3}{c|}{ ANN 2 (Swelling amplitude) } \\
\cline { 2 - 7 } & Training & Validation & Testing & Training & Validation & Testing \\
\hline R & 0.96 & 0.93 & 0.88 & 0.95 & 0.92 & 0.85 \\
\hline MSE & 4.79 & 3.84 & 6.95 & 0.018 & 0.035 & 0.064 \\
\hline
\end{tabular}

\subsection{Validation and test of developed models}

For the data used for the validation and testing steps of the two developed models, the obtained results are almost perfect for both swelling parameters.

For swelling pressure, the values coefficient of correlation (R) and mean square error (MSE) from validation and testing set in ANN 1 were found as 0.93, 3.84 and 0.88, 6.95 respectively as seen in figure 8. For swelling amplitude, the ANN 2 estimates a correlation coefficient (R) and mean square error (MSE) of 0.92 and 0.035 for validation and of 0.85 and 0.064 for testing (Fig.10).

Training: $\mathbf{R}=\mathbf{0} .96725$

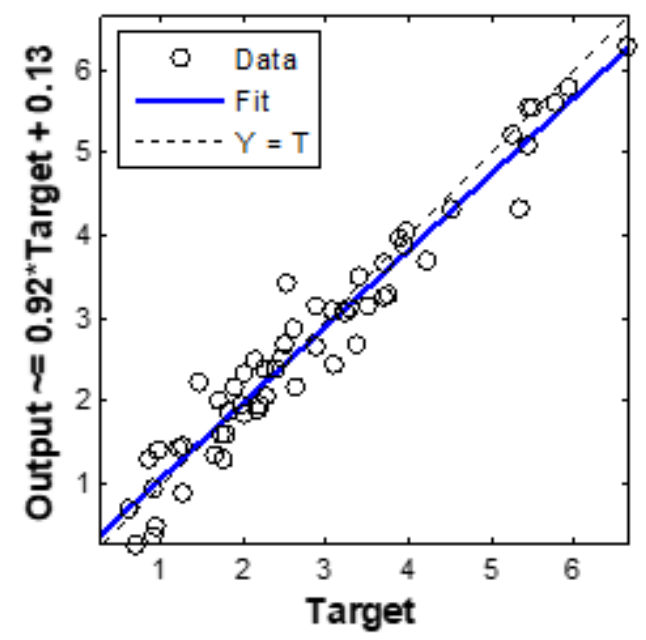

Test: $\mathbf{R}=0.88301$

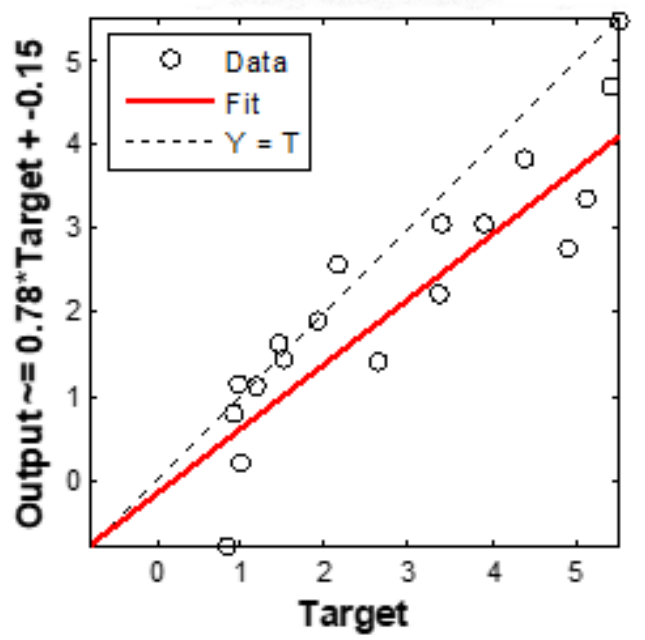

Validation: $\mathbf{R}=0.9373$

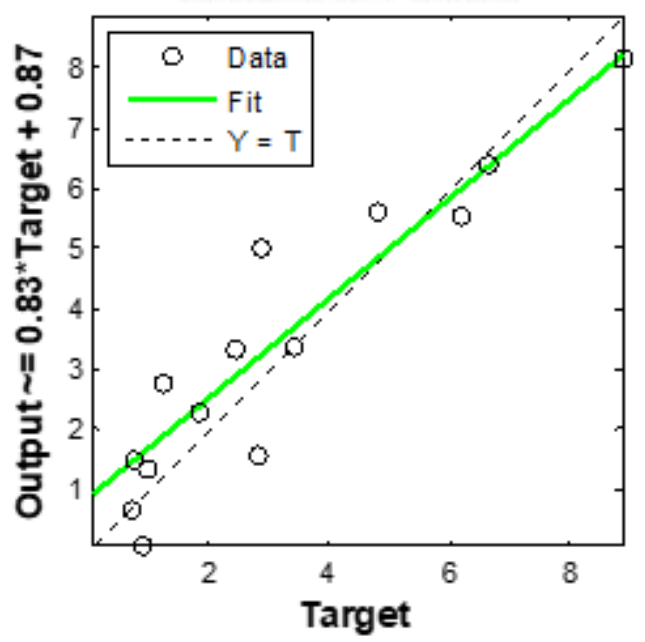

All: $\mathrm{R}=0.92918$

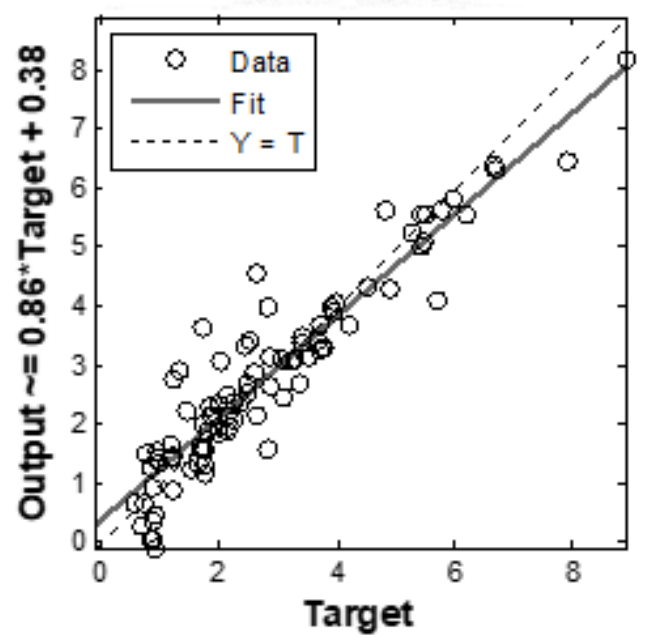

Fig. 8 - Training, Validation and Test of the ANN 1 model for estimating swelling pressure 


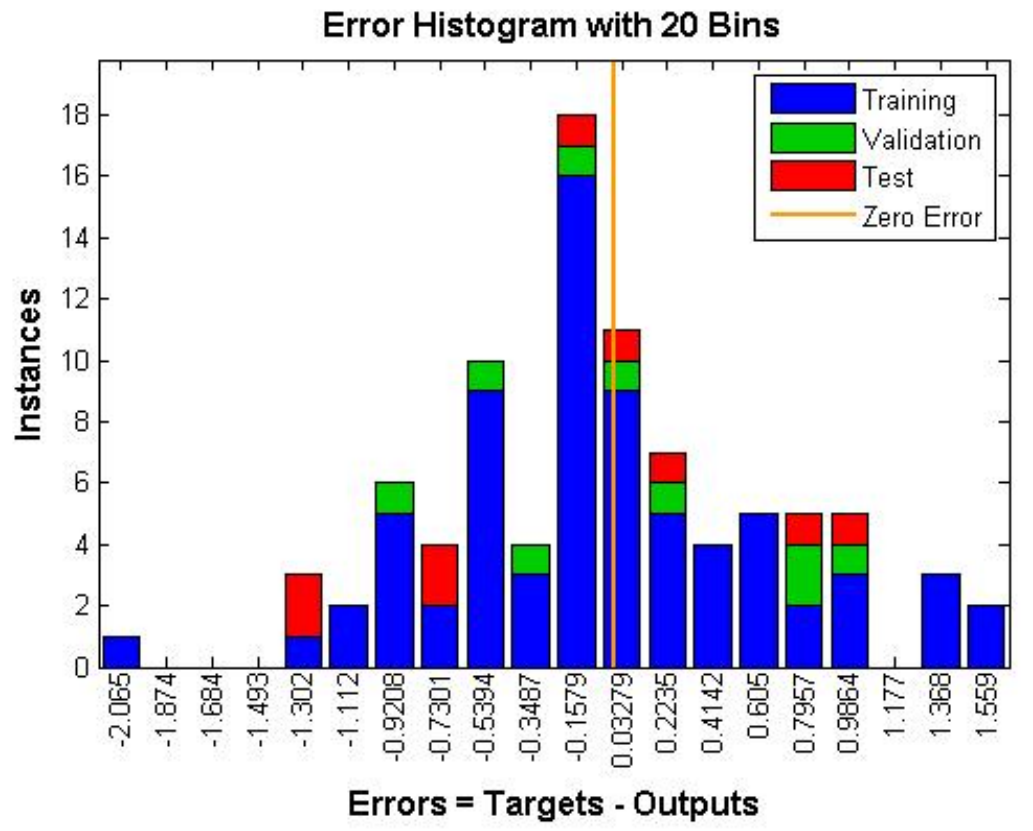

Fig. 9 - Errors results between targets and outputs for swelling pressure

The resulting model is written as in equation 6 :

Output $=Y=b 2+L W \times \tanh (b 1+I W x X)$;

Where:

$$
\begin{aligned}
& \mathrm{b} 1=\text { net.b }\{1\}= \\
& \left(\begin{array}{c}
-1.2035 \\
-1.2436 \\
1.9756 \\
-0.6244 \\
-0.2664 \\
0.7882 \\
-0.4898 \\
1.8043
\end{array}\right) \\
& \mathrm{b} 2=\text { net.b }\{2\}=\quad[-0.5591] \\
& \mathrm{IW}=\operatorname{net.IW}\{1,1\}=\left(\begin{array}{rrrrrrr}
1.0899 & 0.7595 & 1.2295 & -0.0204 & 1.6390 & 0.2143 & -0.9365 \\
0.3928 & -0.4123 & 0.4403 & 0.7275 & 1.1471 & -1.1596 & 1.0033 \\
1.5864 & 2.2928 & -0.9620 & -0.8038 & 0.2992 & -1.9316 & -0.1817 \\
1.3283 & 0.8099 & -0.4464 & 0.6659 & -0.2023 & 0.9781 & 0.3683 \\
-0.1246 & 0.0693 & -1.3103 & 0.1370 & 1.3769 & -0.6902 & 0.7584 \\
-0.9090 & -1.1711 & -0.1005 & 0.7263 & -0.2349 & 0.5652 & -0.8565 \\
-0.2867 & -0.5950 & 0.2577 & 1.5141 & 1.1440 & 0.1505 & 1.9128 \\
1.1026 & -0.6123 & 1.0680 & 0.3120 & 0.0449 & -0.4476 & 0.3157
\end{array}\right)
\end{aligned}
$$

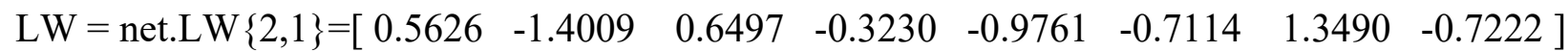


Training: $\mathrm{R}=0.95602$

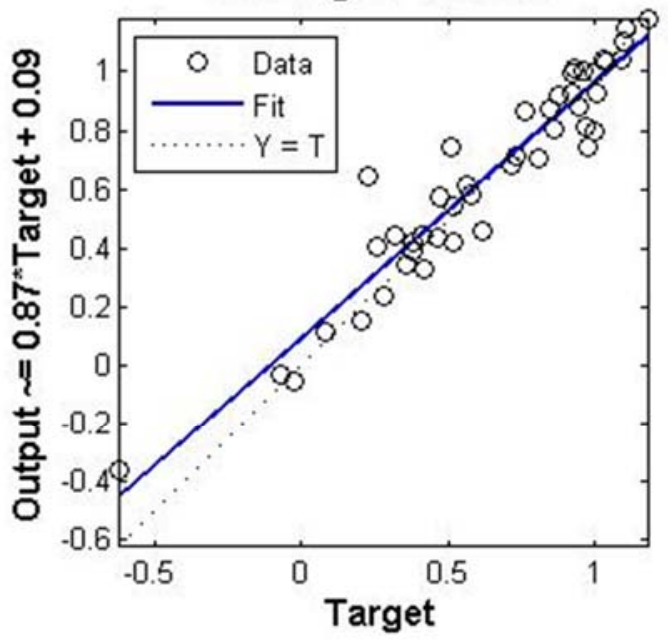

Test: $\mathrm{R}=0.85506$

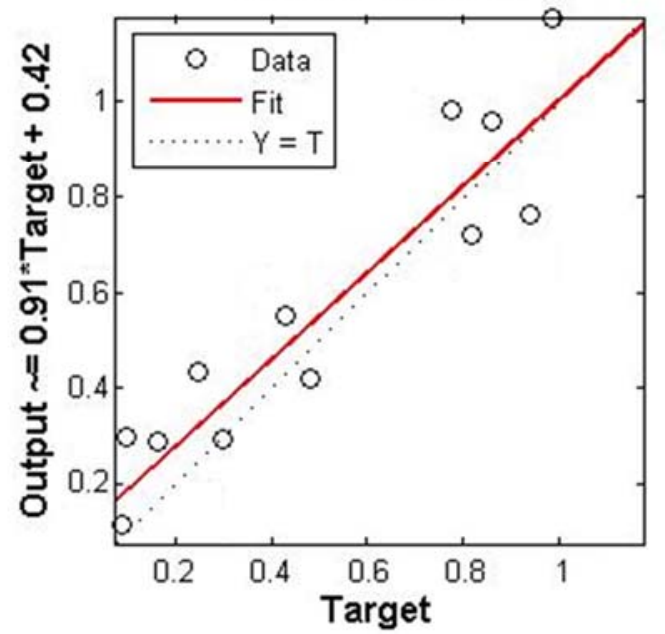

Validation: $\mathrm{R}=0.9265$

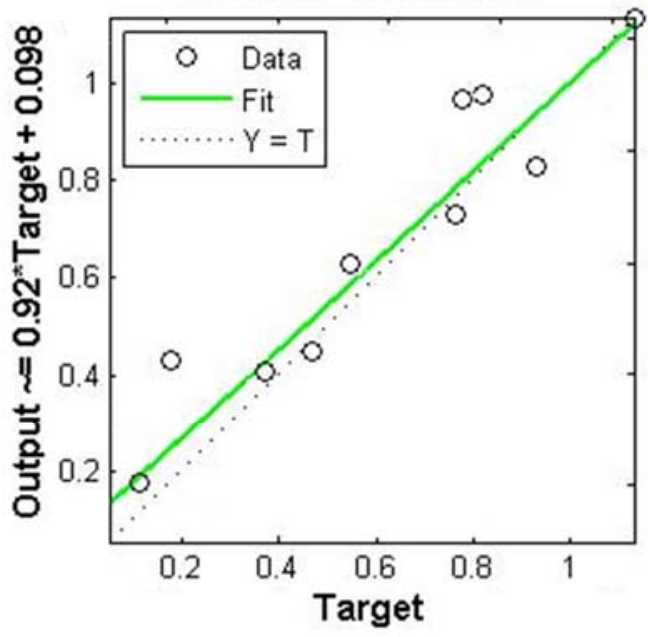

All: $\mathrm{R}=0.91252$

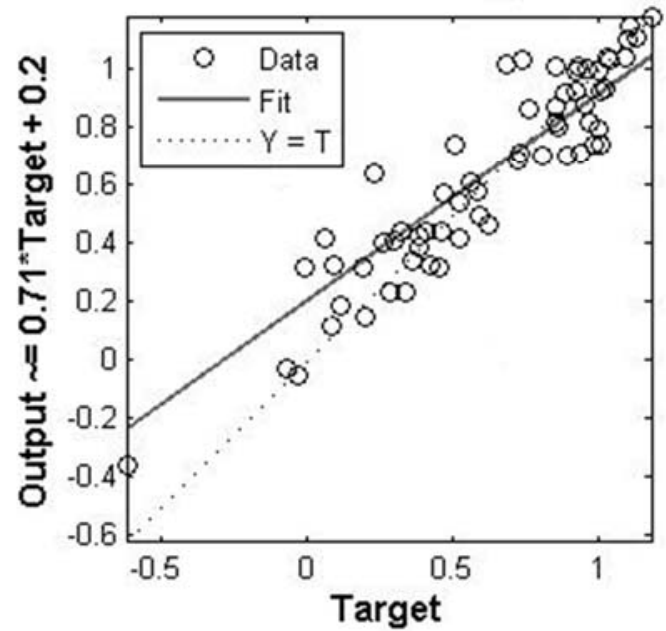

Fig. 10 - Training, Validation and Test of the ANN 2 model for estimating the swelling amplitude

\section{Error Histogram with 20 Bins}

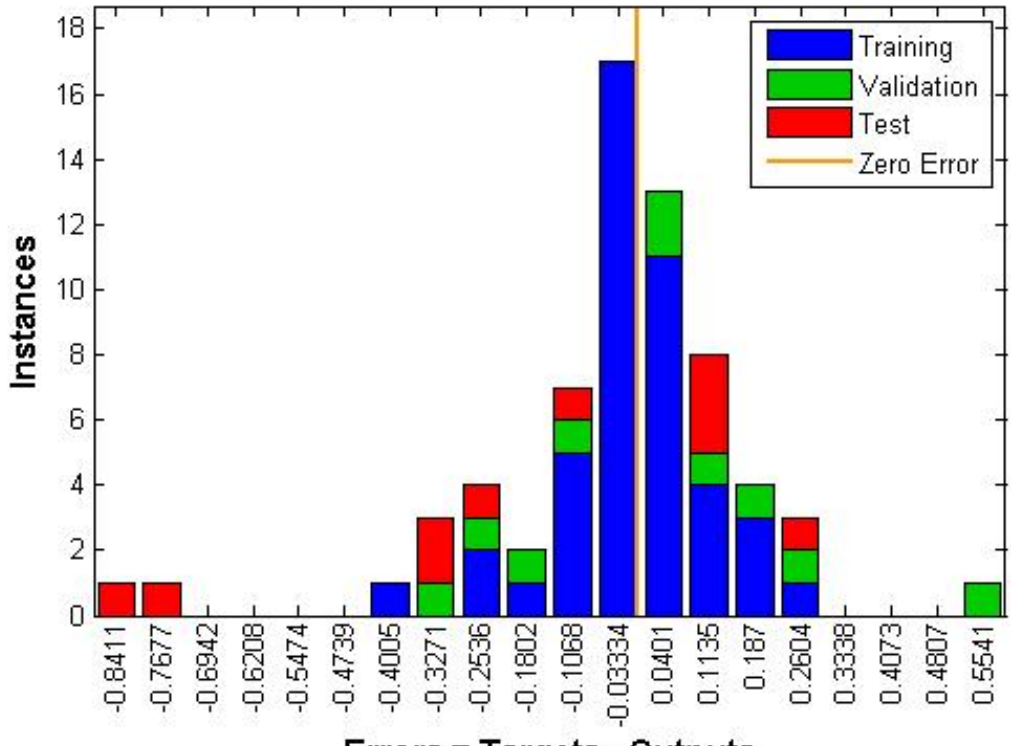

Errors $\mathbf{~}$ Targets $\boldsymbol{~}$ Outputs

Fig. 11 - Errors results between targets and outputs for swelling amplitude 
The resulting model is written as follows:

Output $=Y=b 2+L W \times \tanh (b 1+I W \times X)$;

where :

$$
\begin{aligned}
& \mathrm{b} 1=\text { net.b }\{1\}=\left(\begin{array}{c}
1.7314 \\
-1.3623 \\
-0.6479 \\
\end{array}\right] \\
& \mathrm{b} 2=\text { net.b }\{2\}=[0.8212] \\
& \mathrm{IW}=\text { net.IW }\{1,1\}=\left(\begin{array}{ccccccc}
-0.9548 & -0.8313 & 1.1447 & 0.3502 & 1.1063 & 0.0097 & -0.5818 \\
1.1075 & 0.2075 & -0.9515 & -1.4053 & 0.2928 & 0.2123 & -0.5234 \\
0.5426 & -0.4923 & 0.4791 & 0.1872 & 1.1964 & -0.1515 & 1.1180 \\
& & & & & &
\end{array}\right] \\
& \mathrm{LW}=\text { net.LW }\{2,1\}=\left[\begin{array}{llllll}
0.7278 & 0.8342 & 0.2733
\end{array}\right]
\end{aligned}
$$

\section{Results}

The results obtained by prediction models using artificial neural networks give the following observations:

The first model ANN 1 is highly representative; the values obtained are quite close to those measured with a global correlation coefficient equal to $92.91 \%$.

For the ANN 2 model, the results are also reliable $(\mathrm{R}=91.25 \%)$ with only small discrepancies observed between the direct measurements and the calculations.

The use of ANN significantly improved the estimation of swelling parameters relative to multiple linear regression with an increase in prediction quality of $51.10 \%$ for pressure and $33.47 \%$ for amplitude.

The efficacy of the developed models by ANN in this study for predicting swelling parameters of expansive soils is much better than those developed by MLR. This performance has been demonstrated by many geotechnical researches for the estimation of swelling parameters [25, 26] and by other civil and environmental engineering works for the estimation of various parameters [38-45].

The models obtained by ANN can be used to obtain approximate values of the swelling parameters of sites with similar physical properties.

\section{Conclusion}

Empirical models are indirect techniques for predicting the swelling potential of a soil. These techniques are used for the identification and classification of swelling soils.

The development of multiple linear regression models for estimating the swelling parameters of the two clays of north-western Algeria (Mascara and Tlemcen) was made.

The results showed that prediction models based on statistical analysis can be used satisfactorily to obtain the swelling pressure and swelling amplitude of the studied soils and can reduce the number of direct swelling tests to be performed during the extended reconnaissance phase. 
Artificial neural networks are methods of approximating complex systems, particularly useful when these systems are difficult to model, using conventional statistical methods.

The existence of a model able to consider all the aspects of the behaviour of a soil would be very complex.

However, the applicability of neural networks has been studied for the prediction of swelling parameters using the measured data of our clays. The predictive ability of these models has been tested. The results obtained show a great interest in the use of neural networks for estimating swelling parameters. Thus, their use can help solve complex problems and reduce the number of laboratory tests so that cost and time are saved.

\section{References}

[1] Altmeyer, W.T. (1955). Discussion of engineering properties of expansive clays. Soil Mechanics and Foundations Division. American Society of Testing and Materials. 81(SM2), 17-19.

[2] Seed H.B., Woodward R.J. \& Lundgren R. (1962). Prediction of swelling potential for compacted clays. Proc., A.S.C.E.J. of Soil Mech. and Found. Div. 88(SM3), 53-87.

[3] Ranganatham B.V. \& Satyanarayan B. (1965). A rational method of predicting swelling potential for compacted expansive clays. Proc. 6th Inter. Conf. Soil Mechanics Foundation Engng. 1, 92-96.

[4] Komornik A. \& David D. (1969). Prediction of swelling pressure of clays. J. ASCE, Soil Mechanics and Foundation Division. 1, 209-225.

[5] Vijayvergiya V.N. \& Ghazzaly O.I. (1973). Prediction of swelling potential of natural clays. Proceedings. 3rd Int. Research. And Engng. Conf on Expansive Clays. 227-234.

[6] Dakshanamurthy V. \& Raman V. (1973). A simple method of identifying an expansive soil. Soils and foundations. Japanese Society of Soil Mechanics and Foundation Engineering. 13(1), 97-104.

[7] Williams A.B. \& Donaldson G.W. (1980). Developments related to building on expansive soils in South Africa. Proceedings of the 4th International Conference on Soil Mechanics and Foundations Engineering, Denver. 2, 834-844.

[8] Building Research Establishment. (1980). Low-rise buildings on shrinkable clay soil. BRE, Part 1. BRE Digest 240, HMSO. London.

[9] Snethen, D.R. (1984). Evaluation of expedient methods for identification and classification of potentially expansive soils. Proceedings, 5th International Conference on Expansive Soils. Adelaide. p 22-26.

[10] Chen, F.H. (1988). Foundations on Expansive Soils. Elsevier. p 280. Amsterdam.

[11] Basma, A.A. (1993). Prediction of Expansion Degree for Natural Compacted Clays. Geotechnical Testing Journal. 16(4), 542-549. DOI :10.1520/GTJ10294J.

[12] Al-Shayea, N.A. (2001). The combined effect of clay and moisture content on the behaviour of remodelled unsaturated soil. Engineering Geology. 62, 319-342.

[13] Yilmaz, I. (2006). Indirect estimation of the swelling percent and a new classification of soils depending on liquid limit and cation exchange capacity. Elsevier, Engineering Geology. 85, 295-301. DOI: 10.1016/j.enggeo.2006.02.005

[14]Djedid A., Bekkouche A. \& Aissa Mamoune S.M. (2001). Identification and prediction of swelling of some soils of Tlemcen (Algeria). Bulletin Des Laboratoires des Ponts et Chaussées. №233, 67-75.

[15] Ömür Ç., Nilay K.S. \& Hüseyin Y. (2012). Prediction of Swelling Potential and Pressure in Compacted Clay. Arab J Sci Eng. 37, 1535-1546. DOI : 10.1007/s13369-012-0268-4.

[16] Najjar Y.M. \& Basheer I.A. (1998). Modelling of soil swelling via regression and neural network approaches. Final report No. KS 98-1. Kansas Department of Transportation. 37.

[17] Najjar Y.M., Basheer I.A. \& Mcreynolds R. (1996a). Neural modelling of Kansan soil swelling. Transportation Research Record. 1526, 14-19.

[18] Basma A.A., Barakat S.A. \& Omar M. (2003). Modelling time dependent swell of clays using sequential artificial neural networks. Environ Eng Geosci. 9(3), 279-288.

[19] Meisina C. \& Najjar Y. (2004). An artificial neural network approach for predicting swelling/shrinking parameters of Italian clay soils. European geosciences union, geophysical research abstracts. p. 6.

[20] Moosavi M., Yazdanpanah M.J. \& Doostmohammadi R. (2006). Modelling the cyclic swelling pressure of mudrock using artificial neural networks. Engineering Geology. 87, 178-194. DOI: 10.1016/j.enggeo.2006.07.001.

[21] Erzin, Y. (2007). Artificial neural networks approach for swell pressure versus soil suction behaviour. Canadian Geotechnical Journal. 44(10), 1215-1223. DOI :10.1139/T07-052.

[22] Doris J.J., Rizzo M.D. \& Dewoolkar M.M. (2008). Forecasting vertical ground surface movement from shrinking/swelling soils with artificial neural networks. Int J Numer Anal Methods Geomech. 32, $1229-1245$. DOI: $10.1002 /$ nag. 666 . 
[23] Bekkouche A. \& Aissa Mamoune S.M. (2008). Estimation of swelling parameters using neural networks. GEODIM 08, Saint-Nazaire, France.

[24] Ashayeri I. \& Yasrebi S. (2009). Free-swell and swelling pressure of unsaturated compacted clays; experiments and neural networks. Geotech Geol Eng. 27(1), 137-153. DOI: 10.1007/s10706-008-9219-y.

[25] Aissa Mamoune, S.M. (2009). Characterization and modelling of the clays of Tlemcen using neural networks. Doctoral Thesis, University Abou bakr Belkaid, Tlemcen, Algeria.

[26] Banu Ikizler S., Aytekin M., Vekli M. \& Kocabas F. (2010). Prediction of swelling pressures of expansive soils using artificial neural networks. Advances in Engineering Software. 41, 647-655. DOI: 10.1016/j.advengsoft.2009.12.005

[27] Derriche Z. \& Kebaili M. (1998). Prediction of swelling of in-Aménas clays. Bulletin Des Laboratoires des Ponts et Chaussées. $\mathrm{N}^{\circ} 218,15-23$.

[28] Mobarek, H. (2004). Identification and forecast of the swelling of some clays of Algeria. Magister's thesis, University Mouloud Mammeri, Tizi-Ouzou, Algeria.

[29] Hachichi A. \& Fleureau J.M. (1999). Characterization and stabilization of a few expansive soils from Algeria. Revue Française de Géotechnique, Presses des Ponts et Chaussées. 86, 37-51.

[30] Bahar R. \& Kenai S. (2002). Damages due to water infiltration case of El-Affroun town. Revue Française de Géotechnique, Presses des Ponts et Chaussées. 101, 91-103.

[31] Nayak N.V. \& Christensen R.W. (1971). Swelling characteristics of compacted expansive soils. Clays and Minerals. 19, 251-261.

[32] Weston, D.J. (1980). Expansive Roadbed Treatment for Southern Africa. In $4^{\text {th }}$ International Conference on Expansive Soils, June 16-18 (339-360). Denver, Colorado, United States.

[33] Alayat H., El Badaoui H., Abdallaoui A., Abrid D. \& El Hmaidi A. (2018). Development of mathematical models for predicting the iron concentrations of lake Oubeira waters (ne Algerian). J Fundam Appl Sci. 10(1), 83-96. DOI: $10.4314 /$ jfas.v10i1.6.

[34]Didier, G. (1972). Crystalline and macroscopic swelling of montmorillonites. Thesis for Obtaining the title of Doctor-engineer, University Claude Bernard, France.

[35] Schmitt A., Le Blanc B., Corsini M.M., Lafond C. \& Bruzek J. (2001). Les réseaux de neurones artificiels : Un outil de traitement de données prometteur pour l'anthropologie. Bull. et Mém. de la Société d'Anthropologie de Paris. 13(2), 143-150.

[36] Touzet, C. (1992). Les réseaux de neurones-Artificiels Introduction au Connexionnisme-Cours, exercices et travaux pratiques. page 6.

[37] Shahin M.A., Jaksa M.B. \& Maier H.R. (2001). Artificial neural network applications in geotechnical engineering. Australian Geomechanics Journal. 36(1), 49-62.

[38] Ghiassian H., Jamshidi R. \& Poorebrahim G. (2006). Neural network analysis of silty sand reinforced by carpet wastes. Kuwait.J.Sci.Eng. 33(1), 119-139.

[39] Teh C.I., Wong K.S., Goh A.T.C. \& Jaritngam S. (1997). Prediction of pile capacity using neural networks. J Comput Civil Eng ASCE. 11(2), 129-138.

[40] El Badaoui H., Abdallaoui A., Manssouri I. \& Lancelot L. (2012). Development of Stochastic Mathematical Models for the Prediction of Heavy Metal Levels in Surface Water Using Artificial Neural Networks and Multiple Linear Regression. Journal of Hydrocarbons Mines and Environmental Research. 3, 31-36. DOI: 10.4314/jfas.v10i1.6.

[41]EL Hmaidi A., El Badaoui H., Abdallaoui A. \& Bouchta E. (2013). Application of PMC Artificial Neural Networks for the Prediction of Organic Carbon Content in the Quaternary Terminal Deposits of the Alboran Sea. European Journal of Scientific Research. 107, 400-413.

[42] Kisi, O. (2008). Daily pan evaporation modelling using multi-layer perceptrons and radial basis neural networks. Hydrol Process. 23(2), 213-223. DOI: 10.1002/hyp.7126

[43] El Badaoui H., Abdallaoui A. \& Chabaa S. (2017). Study of the ANN model performance criteria for the prediction of time series humidity, International Journal of Advances in Science Engineering and Technology. (5)1, 117-124.

[44] Bélanger M., El-Jabi N., Caissie D., Ashkar F. \& Ribi J. M. (2005). Estimation de la température de l'eau en rivière en utilisant les réseaux de neurones et la régression linéaire multiple. Revue des sciences de l'eau. 18, 403421. DOI : 10.7202/705565ar.

[45] Bachir R., Aissa Mamoune S.M. \& Habib T. (2018). Using Artificial Neural Networks Approach to Estimate Compressive Strength for Rubberized Concrete. Periodica Polytechnica Civil Engineering. DOI:10.3311/PPci.11928. 